\title{
Some Equivalent Forms of the Arithematic-Geometric Mean Inequality in Probability: A Survey
}

\author{
Cheh-Chih Yeh, ${ }^{1}$ Hung-Wen Yeh, ${ }^{2}$ and Wenyaw Chan $^{3}$ \\ ${ }^{1}$ Department of Information Management, Lunghwa University of Science and Technology, \\ Kueishan Taoyuan, Taoyuan County 33306, Taiwan \\ ${ }^{2}$ Department of Biostatistics, University of Kansas, Kansas City, KS 66160, USA \\ ${ }^{3}$ Division of Biostatistics, University of Texas-Health Science Center at Houston, Houston, \\ TX 77030, USA
}

Correspondence should be addressed to Cheh-Chih Yeh, chehchihyeh@yahoo.com.tw

Received 5 December 2007; Revised 10 April 2008; Accepted 24 June 2008

Recommended by Jewgeni Dshalalow

We link some equivalent forms of the arithmetic-geometric mean inequality in probability and mathematical statistics.

Copyright (c) 2008 Cheh-Chih Yeh et al. This is an open access article distributed under the Creative Commons Attribution License, which permits unrestricted use, distribution, and reproduction in any medium, provided the original work is properly cited.

\section{Introduction}

The arithmetic-geometric mean inequality (in short, AG inequality) has been widely used in mathematics and in its applications. A large number of its equivalent forms have also been developed in several areas of mathematics. For probability and mathematical statistics, the equivalent forms of the AG inequality have not been linked together in a formal way. The purpose of this paper is to prove that the AG inequality is equivalent to some other renowned inequalities by using probabilistic arguments. Among such inequalities are those of Jensen, Hölder, Cauchy, Minkowski, and Lyapunov, to name just a few.

\section{The equivalent forms}

Let $X$ be a random variable, we define

$$
E_{r}|X|:= \begin{cases}\left(E|X|^{r}\right)^{1 / r}, & \text { if } r \neq 0, \\ \exp (E(\ln |X|)), & \text { if } r=0\end{cases}
$$

where EX denotes the expected value of $X$. 
Throughout this paper, let $n$ be a positive integer and we consider only the random variables which have finite expected values.

In order to establish our main results, we need the following lemma which is due to Infantozzi [1, 2], Marshall and Olkin [3, Page 457], and Maligranda [4, 5]. For other related results, we refer to [6-19].

Lemma 2.1. The following inequalities are equivalent.

$\left(E_{1}\right)$ AG inequality: $E X \geq e^{E \ln X}$, where $X$ is a nonnegative random variable.

$\left(E_{2}\right) a_{1}^{q_{1}} a_{2}^{q_{2}} \cdots a_{n}^{q_{n}} \leq a_{1} q_{1}+a_{2} q_{2}+\cdots+a_{n} q_{n}$ if $a_{i} \in(0, \infty)$ and $q_{i} \in(0,1)$ for $i=1,2, \ldots, n$ with $\sum_{i=1}^{n} q_{i}=1$. The arithmetic-geometric mean inequality is usually applied in a simple version of $\left(E_{2}\right)$ with $q_{i}=1 / n$ for each $i=1,2, \ldots, n$.

$\left(E_{3}\right) a^{\alpha} b^{1-\alpha} \leq \alpha a+(1-\alpha) b$ if $0<\alpha<1$ and $a, b>0$, and the opposite inequality holds if $\alpha>1$ or $\alpha<0$.

$\left(E_{4}\right)(y+1)^{\alpha}<1+\alpha y$ if $0<\alpha<1$ and $y>-1$, and the opposite inequality holds if $\alpha>1$ or $\alpha<0$ and $y>-1$.

$\left(E_{5}\right) \sum_{i=1}^{n} a_{i}^{p} b_{i}^{q} \leq\left(\sum_{i=1}^{n} a_{i}\right)^{p}\left(\sum_{i=1}^{n} b_{i}\right)^{q}$ for $a_{i}, b_{i} \in(0, \infty), i=1,2, \ldots, n$ if $p>0, q>0$ with $p+q \geq 1$, and the opposite inequality holds if $p q<0$ with $p+q \leq 1$.

$\left(E_{6}\right)\left[\sum_{i=1}^{n}\left(a_{i}+b_{i}\right)^{p}\right]^{1 / p} \geq\left(\sum_{i=1}^{n} a_{i}^{p}\right)^{1 / p}+\left(\sum_{i=1}^{n} b_{i}^{p}\right)^{1 / p}$ if $p \leq 1$ and $a_{i}, b_{i} \in(0, \infty)$ for $i=$ $1,2, \ldots, n$, and the opposite inequality holds if $p \geq 1$.

$r<s<t$.

(E) $\left(\sum_{i=1}^{n} a_{i} b_{i}^{s}\right)^{t-r} \leq\left(\sum_{i=1}^{n} a_{i} b_{i}^{r}\right)^{t-s}\left(\sum_{i=1}^{n} a_{i} b_{i}^{t}\right)^{s-r}$ if $a_{i}, b_{i} \in(0, \infty)$ for $i=1,2, \ldots, n$ and

$\left(E_{8}\right)$ Let $(\Omega, \boldsymbol{B}, \mu)$ be a measure space. If $f_{i}: \Omega \rightarrow[0, \infty)$ is finitely $\mu$-integrable, $i=1,2, \ldots, n$ and let $q_{i} \geq 0, \sum_{i=1}^{n} q_{i}=1$. Then $\prod_{i=1}^{n} f_{i}^{q_{i}}$ is finitely integrable and $\int \prod_{i=1}^{n} f_{i}^{q_{i}} d \mu \leq \prod_{i=1}^{n}\left(\int f_{i}^{q_{i}} d \mu\right)$.

$\left(E_{9}\right)$ If $a \geq b \geq c$ and $f: \Omega \rightarrow \mathbb{R}$ is $\mu$-integrable, where $(\Omega, \mathbb{B}, \mu)$ is a probability space, then $\left(\int|f|^{b} d \mu\right)^{a-c} \leq\left(\int|f|^{c} d \mu\right)^{a-b}\left(\int|f|^{a} d \mu\right)^{b-c}$.

$\left(E_{10}\right)$ Artin's theorem. Let $K$ be an open convex subset of $\mathbb{R}$ and $f: K \times(a, b) \rightarrow[0, \infty)$ satisfy

(a) $f(x, y)$ is Borel-measurable in $y$ for each fixed $x$,

(b) $\log f(x, y)$ is convex in $x$ for each fixed $y$.

If $\mu$ is a measure on the Borel subsets of $(a, b)$ such that $f(x, \cdot)$ is $\mu$-integrable for each $x \in K$, then $g(x):=\log \int_{a}^{b} f(x, y) d \mu(y)$ is a convex function on $K$.

$\left(E_{11}\right)$ Jensen's inequality. Let $\Omega$ be a probability space and $X$ be a random variable taking values in the open convex set $A \subset \mathbb{R}$ with finite expectation $E X$. If $f: A \rightarrow \mathbb{R}$ is convex, then $E f(X) \geq$ $f(E X)$.

Proof. The proof of the equivalent relations of $\left(E_{2}\right),\left(E_{3}\right),\left(E_{4}\right), \ldots,\left(E_{7}\right)$ can be found in $[1,2$, $4,5]$.

The proof of the equivalent relations of $\left(E_{1}\right),\left(E_{2}\right),\left(E_{8}\right),\left(E_{9}\right),\left(E_{10}\right)$, and $\left(E_{11}\right)$ can be found in [3].

Theorem 2.2. The following inequalities are equivalent.

$\left(H_{0}\right) E|X Y| \leq\left(E_{p}|X|\right)\left(E_{q}|Y|\right)$ if $X, Y$ are random variables and $1 / p+1 / q=1$ with $p>1$ and $q>1$.

$\left(H_{1}\right) E|Z||X|^{h}|Y|^{k} \leq(E|Z||X|)^{h}(E|Z||Y|)^{k}$ if $X, Y, Z$ are random variables and $h+k=1$ with $h>0$ and $k>0$.

$\left(H_{2}\right) E|Z||X|^{h}|Y|^{k} \geq(E|Z||X|)^{h}(E|Z||Y|)^{k}$ if $X, Y, Z$ are random variables and $h+k=1$ with $h k<0$. 
$\left(H_{2}^{*}\right) E|X|^{h}|Y|^{k} \geq(E|X|)^{h}(E|Y|)^{k}$ if $X, Y$ are random variables and $h+k=1$ with $h k<0$, that is, $E|X Y| \geq\left(E_{p}|X|\right)\left(E_{q}|Y|\right)$ if $1 / p+1 / q=1$ with $0<p<1$. and $k>0$.

$\left(H_{3}\right) E|X|^{h}|Y|^{k} \leq(E|X|)^{h}(E|Y|)^{k}$ if $X, Y$ are random variables and $h+k \leq 1$ with $h>0$

$\left(H_{4}\right) E|X|^{h}|Y|^{k} \geq(E|X|)^{h}(E|Y|)^{k}$ if $X, Y$ are random variables and $h+k \geq 1$ with $h k<0$.

$\left(L_{1}\right)\left(E|Z||X|^{s}\right)^{t-r} \leq\left(E|Z||X|^{t}\right)^{s-r}\left(E|Z||X|^{r}\right)^{t-s}$ if $X, Z$ are random variables and $r<s<t$.

$\left(L_{2}\right)\left(E|Z||X|^{s}\right)^{t-r} \geq\left(E|Z||X|^{t}\right)^{s-r}\left(E|Z||X|^{r}\right)^{t-s}$ if $X, Z$ are random variables and $s<r<t$.

$\left(L_{3}\right)\left(E|X|^{r}\right)^{1 / r} \leq\left(E|X|^{s}\right)^{1 / s}$ if $X$ is a random variable and $r \leq s$, that is, $\left(E|X|^{r}\right)^{1 / r}$ is nondecreasing on $r$.

$\left(L_{4}\right)($ see $[10,18])\left(E|X|^{p}\right) \geq(E|X|)^{p}$, where $X$ is a random variable if $p \geq 1$ or $p \leq 0$, and the opposite inequality holds if $0 \leq p \leq 1$.

$\left(R_{1}\right)\left(E|X|^{r}\right)^{p} /\left(E|Y|^{r}\right)^{q} \leq E\left(|X|^{p} /|Y|^{q}\right)^{r}$ if $X, Y$ are random variables and $p \geq q+r$ with $p>0, q>0, r>0$.

$\left(R_{2}\right)\left(E|X|^{r}\right)^{p} /\left(E|Y|^{r}\right)^{q} \leq E\left(|X|^{p} /|Y|^{q}\right)^{r}$ if $X, Y$ are random variables and $p \geq q+r$ with $p<0, q<0, r>0$.

$\left(R_{3}\right)\left(E|X|^{r}\right)^{p} /\left(E|Y|^{r}\right)^{q} \geq E\left(|X|^{p} /|Y|^{q}\right)^{r}$ if $X, Y$ are random variables and $p \leq q+r$ with $p>0, q<0, r>0$.

$\left(R_{4}\right)(E|X|)^{p} /(E|Y|)^{p-1} \leq E\left(|X|^{p} /|Y|^{p-1}\right)$ if $X, Y$ are random variables and $p \geq 1$.

$\left(R_{5}\right)(E|X|)^{p} /(E|Y|)^{p-1} \leq E\left(|X|^{p} /|Y|^{p-1}\right)$ if $X, Y$ are random variables and $p<0$.

$\left(R_{6}\right)(E|X|)^{p} /(E|Y|)^{p-1} \geq E\left(|X|^{p} /|Y|^{p-1}\right)$ if $X, Y$ are random variables and $0<p<1$.

$\left(C_{1}\right)$ Cauchy-Bunyakouski and Schwarz's (CBS) inequality: $(E|X Y|)^{2} \leq\left(E|X|^{2}\right)\left(E|Y|^{2}\right)$ if $X, Y$ are random variables.

$\left(C_{1}^{*}\right)(E|X Y Z|)^{2} \leq\left(E|Z||X|^{2}\right)\left(E|Z||Y|^{2}\right)$ if $X, Y, Z$ are random variables.

$\left(C_{2}\right)\left[E\left(Z|X|^{s}|Y|^{1-s}\right)\right]\left[E\left(Z|X|^{1-s}|Y|^{s}\right)\right] \leq(E|Z||X|)(E|Z||Y|)$ if $X, Y, Z$ are random variables and $s \in(0,1)$ (the inequality is reversed if $s>1$ or $s<0)$.

$\left(C_{3}\right)\left[E\left(|Z||X|^{p+r}|Y|^{p-r}\right)\right]\left[E\left(|Z||X|^{p-r}|Y|^{p+r}\right)\right] \leq\left[E\left(|Z||X|^{p+s}|Y|^{p-s}\right)\right]\left[E\left(|Z||X|^{p-s}|Y|^{p+s}\right)\right]$ for any $p \in \mathbb{R}$ if $X, Y, Z$ are random variables and $|r| \leq|s|$.

$\left(C_{4}\right)\left[E\left(|Z||X|^{r}|Y|^{s}\right)\right]\left[E\left(|Z||X|^{s}|Y|^{r}\right)\right] \leq\left[E\left(|Z||X|^{u}|Y|^{v}\right)\right]\left[E\left(|Z||X|^{v}|Y|^{u}\right)\right]$ if $X, Y, Z$ are random variables and either $0 \leq v \leq s \leq r \leq u, r+s=u+v$ or $0 \leq u \leq r \leq s \leq v, r+s=u+v$. $|r| \leq|s|$.

$\left(C_{5}\right)\left[E|Z||X|^{r}\right]\left[E|Z||X|^{-r}\right] \leq\left[E|Z||X|^{s}\right]\left[E\left(|Z||X|^{-s}\right]\right.$ if $X, Y, Z$ are random variables and

$\left(C_{6}\right)\left[E\left(|Z||X|^{p-s}|Y|^{s}\right)\right]\left[E\left(|Z||X|^{s}|Y|^{p-s}\right)\right] \leq\left[E|Z||X|^{p-r}|Y|^{r}\right]\left[E|Z||X|^{r}|Y|^{p-r}\right]$ if $X, Y, Z$ are random variables and either $p / 2 \leq s \leq r \leq p$ or $0 \leq r \leq s \leq p / 2$.

$\left(C_{7}\right)\left[E\left(|Z||X|^{2-s}|Y|^{s}\right)\right]\left[E\left(|Z||X|^{s}|Y|^{2-s}\right)\right] \leq\left[E|Z||X|^{2-r}|Y|^{r}\right]\left[E|Z||X|^{r}|Y|^{2-r}\right]$ if $X, Y, Z$ are random variables and either $0 \leq r \leq s \leq 1$ or $1 \leq s \leq r \leq 2$.

$\left(C_{8}\right)\left[E|Z||X|^{k+s}|Y|^{l-t}\right]\left[E|Z||X|^{k-s}|Y|^{l+t}\right]$ increases with $|s|$ if $X, Y, Z$ are random variables and $k / l=s / t$.

(M) Minkowski's inequality: $E_{p}|X+Y| \leq E_{p}|X|+E_{p}|Y|$ if $X, Y$ are random variables, $p \geq 1$, and the opposite inequality holds if $p \leq 1$.

(T) Triangle inequality: $E_{p}|X-Y| \leq E_{p}|X-Z|+E_{p}|Z-Y|$ if $X, Y, Z$ are random variables, $p \geq 1$, and the opposite inequality holds if $p \leq 1$.

$\left(J_{1}\right) G_{2} G_{1}^{-1}(E Y) \leq E G_{2} G_{1}^{-1}(Y)$ if $Y$ is a random variable, $G_{1}$ and $G_{2}$ are two continuous and strictly increasing functions such that $G_{2} G_{1}^{-1}$ is convex.

$\left(J_{2}\right) E e^{t X} \geq e^{t E X}$ for any $t \in \mathbb{R}$ if $X$ is a random variable.

The above listed inequalities are also equivalent to the inequalities in Lemma 2.1. 
Proof. The sketch of the proof of this theorem is illustrated by the following maps of equivalent circles:

(1) $\left(E_{3}\right) \Rightarrow\left(H_{0}\right) \Leftrightarrow\left(H_{1}\right) \Leftrightarrow\left(H_{2}\right) \Leftrightarrow\left(H_{2}^{*}\right)$;

(2) $\left(H_{1}\right) \Rightarrow\left(L_{1}\right) \Rightarrow\left(H_{0}\right) \Rightarrow\left(L_{3}\right) \Rightarrow\left(H_{3}\right)$;

(3) $\left(H_{2}\right) \Rightarrow\left(L_{2}\right) \Rightarrow\left(H_{2}^{*}\right) \Rightarrow\left(H_{4}\right)$;

(4) $\left(L_{1}\right) \Rightarrow\left(L_{3}\right) \Leftrightarrow\left(L_{4}\right),\left(L_{2}\right) \Rightarrow\left(L_{3}\right) \Rightarrow\left(E_{1}\right)$;

(5) $\left(H_{3}\right) \Rightarrow\left(R_{1}\right) \Rightarrow\left(R_{4}\right) \Rightarrow\left(H_{2}^{*}\right),\left(H_{4}\right) \Rightarrow\left(R_{2}\right) \Rightarrow\left(R_{5}\right) \Rightarrow\left(H_{2}\right),\left(H_{4}\right) \Rightarrow\left(R_{3}\right) \Rightarrow$ $\left(R_{6}\right) \Rightarrow\left(H_{2}\right)$;

(6) $\left(H_{0}\right) \Leftrightarrow(M) \Leftrightarrow(T)$;

(7) $\left(C_{1}\right) \Rightarrow\left(H_{0}\right) \Rightarrow\left(H_{1}\right) \Rightarrow\left(C_{2}\right) \Rightarrow\left(C_{3}\right) \Rightarrow\left(C_{4}\right) \Rightarrow\left(C_{6}\right) \Rightarrow\left(C_{7}\right) \Rightarrow\left(C_{1}\right) \Leftrightarrow\left(C_{1}^{*}\right)$;

(8) $\left(C_{4}\right) \Rightarrow\left(C_{5}\right) \Rightarrow\left(C_{3}\right) \Leftrightarrow\left(C_{8}\right)$;

(9) $\left(E_{11}\right) \Rightarrow\left(J_{1}\right) \Rightarrow\left(L_{3}\right),\left(E_{11}\right) \Rightarrow\left(J_{2}\right) \Rightarrow\left(E_{1}\right)$.

Now, we are in a position to give the proof of this theorem as follows.

$\left(E_{3}\right) \Rightarrow\left(H_{0}\right)$, see Casella and Berger [7, page 187].

$\left(H_{0}\right) \Leftrightarrow\left(H_{1}\right)$ is clear.

$\left(H_{1}\right) \Rightarrow\left(H_{2}\right)$ : If $h<0$ and $k>0$, then $-k / h>0$ and $-h / k+1 / k=1$. This and $\left(H_{1}\right)$ imply

$$
E|Z||X|^{-h / k}|Y|^{1 / k} \leq(E|Z||X|)^{-h / k}(E|Z||Y|)^{1 / k} .
$$

Replacing $|Y|$ by $|X|^{h}|Y|^{k}$ in the above inequality, we obtain $\left(H_{2}\right)$.

Similarly, we can prove the case that $h>0$ and $k<0$.

$\left(H_{2}\right) \Rightarrow\left(H_{1}\right)$ is proved similarly.

$\left(H_{2}\right) \Leftrightarrow\left(H_{2}^{*}\right)$ is clear.

$\left(H_{1}\right) \Rightarrow\left(L_{1}\right)$. Letting $|X|,|Y|, h$, and $k$ be replaced by $|X|^{t},|X|^{r},(s-r) /(t-r)$ and $(t-s) /(t-r)$ in $\left(H_{1}\right)$, respectively, we obtain $\left(L_{1}\right)$.

$\left(H_{2}\right) \Rightarrow\left(L_{2}\right)$ is similarly proved.

$\left(L_{1}\right) \Rightarrow\left(H_{0}\right)$ : Let $h=(t-s) /(t-r), k=(s-r) /(t-r)$. Then $h+k=1, h>0, k>0$. It follows from $\left(L_{1}\right)$ that

$$
\begin{aligned}
E(|X \| Y|)= & E\left(|X|^{t /(t-s)}|Y|^{-r /(s-r)}\left(|X|^{-1 /(t-s)}|Y|^{1 /(s-r)}\right)^{s}\right) \\
\leq & {\left[E|X|^{t /(t-s)}|Y|^{-r /(s-r)}\left(|X|^{-1 /(t-s)}|Y|^{1 /(s-r)}\right)^{r}\right]^{(t-s) /(t-r)} } \\
& \times\left[E|X|^{t /(t-s)}|Y|^{-r /(s-r)}\left(|X|^{-1 /(t-s)}|Y|^{1 /(s-r)}\right)^{t}\right]^{(s-r) /(t-r)} \\
= & E_{1 / h}|X| E_{1 / k}|Y| .
\end{aligned}
$$

That is, $\left(H_{0}\right)$ holds.

$\left(L_{2}\right) \Rightarrow\left(H_{2}^{*}\right)$ is similarly proved.

$\left(H_{0}\right) \Rightarrow\left(L_{3}\right) \Rightarrow\left(H_{3}\right)$. Taking $Y=1$ in $\left(H_{0}\right)$, we see that

$$
E|X| \leq\left(E|X|^{p}\right)^{1 / p}, \quad p>1,
$$


which implies

$$
(E|X|)^{r / s} \leq E|X|^{r / s}, \quad p=\frac{r}{s} .
$$

Replacing $|X|$ by $|X|^{s}$,

$$
\left(E|X|^{s}\right)^{r / s} \leq E|X|^{r}
$$

Thus

$$
\begin{aligned}
& \left(E|X|^{s}\right)^{1 / s} \leq\left(E|X|^{r}\right)^{1 / r} \quad \text { if } r>s>0 \\
& \left(E|X|^{s}\right)^{1 / s} \geq\left(E|X|^{r}\right)^{1 / r} \quad \text { if } r<s<0
\end{aligned}
$$

This proves $\left(L_{3}\right)$.

Next, let $p=h+k$. Then $h / p+k / p=1$ and $0<p \leq 1$. This and $\left(H_{0}\right)$ imply

$$
E|X|^{h / p}|Y|^{k / p} \leq(E|X|)^{h / p}(E|Y|)^{k / p} .
$$

Replacing $|X|$ and $|Y|$ by $|X|^{p}$ and $|Y|^{p}$ in the above inequality, respectively, and using $\left(L_{3}\right)$, we obtain

$$
E|X|^{h}|Y|^{k} \leq\left(E_{p}|X|\right)^{h}\left(E_{p}|Y|\right)^{k} \leq(E|X|)^{h}(E|Y|)^{k}
$$

This proves $\left(H_{3}\right)$ holds.

$\left(H_{2}^{*}\right) \Rightarrow\left(H_{4}\right)$ is proved similarly.

$\left(L_{1}\right) \Rightarrow\left(L_{3}\right)$. (a) Taking $Z=1$ and $t=0$ in $\left(L_{1}\right)$,

$$
E\left(|X|^{s}\right)^{-r} \leq E\left(|X|^{r}\right)^{-s} \text { if } r<s<0,
$$

which implies

$$
\left(E|X|^{r}\right)^{1 / r} \leq\left(E|X|^{s}\right)^{1 / s} \text { if } r<s<0
$$

(b) Taking $Z=1$ and $r=0$ in $\left(L_{1}\right)$,

$$
\left(E|X|^{s}\right)^{t} \leq\left(E|X|^{t}\right)^{s} \quad \text { if } 0<s<t
$$

which implies

$$
\left(E|X|^{s}\right)^{1 / s} \leq\left(E|X|^{t}\right)^{1 / t} \quad \text { if } 0<s<t .
$$


(c) Taking $Z=1$ and $s=0$ in $\left(L_{1}\right)$,

$$
1 \leq\left(E|X|^{t}\right)^{-r}\left(E|X|^{r}\right)^{t} \quad \text { if } r<0<t,
$$

which implies

$$
\left(E|X|^{r}\right)^{1 / r} \leq\left(E|X|^{t}\right)^{1 / t} \quad \text { if } r<0<t .
$$

(d) It follows from (a), (b), and (c) that $\left(L_{3}\right)$ holds.

Thus, we complete the proof.

$\left(L_{2}\right) \Rightarrow\left(L_{3}\right)$ is similarly proved.

$\left(L_{3}\right) \Rightarrow\left(L_{4}\right)$ is clear.

$\left(L_{4}\right) \Rightarrow\left(L_{3}\right)$ by using the technique of $\left(H_{0}\right) \Rightarrow\left(L_{3}\right)$.

$\left(L_{3}\right) \Rightarrow\left(E_{1}\right)$. Letting $r \rightarrow 0$ and $s=1$ in $\left(L_{3}\right)$, we obtain $\left(E_{1}\right)$.

$\left(H_{3}\right) \Rightarrow\left(R_{1}\right)$. It follows from $p \geq q+r$ and $p, q, r \in(0, \infty)$ that $q / p+r / p \leq 1$. This and $\left(H_{3}\right)$ imply

$$
E|X|^{q / p}|Y|^{r / p} \leq(E|X|)^{q / p}(E|Y|)^{r / p}
$$

Replacing $|X|$ and $|Y|$ by $|Y|^{r}$ and $|X|^{p}|Y|^{-q}$ in the above inequality, respectively, we obtain $\left(R_{1}\right)$.

$\left(H_{4}\right) \Rightarrow\left(R_{2}\right)$ and $\left(H_{4}\right) \Rightarrow\left(R_{3}\right)$ are similarly proved.

$\left(R_{1}\right) \Rightarrow\left(R_{4}\right),\left(R_{2}\right) \Rightarrow\left(R_{5}\right)$ and $\left(R_{3}\right) \Rightarrow\left(R_{6}\right)$ follow by taking $q=p-1$ and $r=1$.

$\left(R_{4}\right) \Rightarrow\left(H_{2}^{*}\right),\left(R_{5}\right) \Rightarrow\left(H_{2}\right)$ and $\left(R_{6}\right) \Rightarrow\left(H_{0}\right)$ follow by taking $p=h, k=1-p$ in $\left(R_{4}\right),\left(R_{5}\right)$ and $\left(R_{6}\right)$, respectively.

$\left(H_{0}\right) \Rightarrow(M)$ Casella and Berger [7, page 188].

$(M) \Rightarrow\left(H_{0}\right)$ (see [5]): Let $1 / p+1 / q=1$ with $p>1$ and $q>1$. It follows from Benoulli's inequality $\left(E_{4}\right)$ that

$$
p t|X||Y| \leq\left(|Y|^{1 /(p-1)}+t|X|\right)^{p}-|Y|^{p /(p-1)}, \quad \text { for } t>0 .
$$

This and $(M)$ imply

$$
p t E|X||Y| \leq\left[\left(E|Y|^{p /(p-1)}\right)^{1 / p}+t\left(E|X|^{p}\right)^{1 / p}\right]^{p}-E|Y|^{p /(p-1)}, \quad \text { for } t>0 .
$$

Hence

$$
\begin{aligned}
p E|X \| Y| & \leq \lim _{t \rightarrow 0^{+}} \inf \frac{1}{t}\left\{\left[\left(E|Y|^{p /(p-1)}\right)^{1 / p}+t\left(E|X|^{p}\right)^{1 / p}\right]^{p}-E|Y|^{p /(p-1)}\right\} \\
& =p E_{p}|X| E_{q}|Y| .
\end{aligned}
$$

This proves $\left(H_{0}\right)$ holds.

$(M) \Rightarrow(T)$ follows by replacing $X$ and $Y$ by $X-Z$ and $Z-Y$ in $(M)$, respectively.

$(T) \Rightarrow(M)$ follows by replacing $Y$ and $Z$ in $(T)$ with $Y$ and 0 , respectively. 
$\left(C_{1}\right) \Rightarrow\left(H_{0}\right)$. Let $F(x)=E|Y|^{q}\left(|X|^{p}|Y|^{-q}\right)^{x}$ for $x \in(0,1)$. Then, it follows from $\left(C_{1}\right)$ that

$$
\begin{aligned}
F\left(\frac{x_{1}}{2}+\frac{x_{2}}{2}\right) & =E\left\{\left[|Y|^{q}\left(|X|^{p}|Y|^{-q}\right)^{x_{1}}\right]^{1 / 2}\left[|Y|^{q}\left(|X|^{p}|Y|^{-q}\right)^{x_{2}}\right]^{1 / 2}\right\} \\
& \leq\left(E|Y|^{q}\left(|X|^{p}|Y|^{-q}\right)^{x_{1}}\right)^{1 / 2}\left(E|Y|^{q}\left(|X|^{p}|Y|^{-q}\right)^{x_{2}}\right)^{1 / 2} \\
& =F\left(x_{1}\right)^{1 / 2}+F\left(x_{2}\right)^{1 / 2} .
\end{aligned}
$$

Thus, $\ln F$ is midconvex on $(0,1)$, and hence $\ln F$ is convex on $(0,1)$. Hence

$$
\ln F\left(\frac{r}{p}+\frac{1-r}{q}\right) \leq \frac{1}{p} \ln F(r)+\frac{1}{q} \ln F(1-r) .
$$

Therefore,

$$
F\left(\frac{r}{p}+\frac{1-r}{q}\right) \leq F^{1 / p}(r) F^{1 / q}(1-r)
$$

Letting $r \rightarrow 1^{-}$in the both sides of the above inequality,

$$
E|X Y|=F\left(\frac{1}{p}\right) \leq F^{1 / p}(1) F^{1 / q}(0)=\left(E_{p}|X|\right)\left(E_{q}|Y|\right)
$$

This shows $\left(H_{0}\right)$ (see [13]).

$\left(H_{1}\right) \Rightarrow\left(C_{2}\right)$. First note that, as shown above, $\left(H_{1}\right)$ and $\left(H_{2}\right)$ are equivalent. It follows from $\left(H_{1}\right)$ that, for $s \in(0,1)$,

$$
\begin{aligned}
& E|Z||X|^{s}|Y|^{1-s} \leq(E|Z||X|)^{s}(E|Z||Y|)^{1-s} \\
& E|Z||X|^{1-s}|Y|^{s} \leq(E|Z||X|)^{1-s}(E|Z||Y|)^{s} .
\end{aligned}
$$

These imply $\left(C_{2}\right)$ for the case $s \in(0,1)$.

Similarly, we can prove the case for $s>1$ or $s<0$ by using $\left(H_{2}\right)$.

$\left(C_{2}\right) \Rightarrow\left(C_{3}\right)$ follows by replacing $s,|X|,|Y|$ in $\left(C_{2}\right)$ by $(1 / 2)(1+r / s)$ if $r s>0$ or $(1 / 2)(1-r / s)$ if $r s<0,|X|^{p+s}|Y|^{p-s},|X|^{p-s}|Y|^{p+s}$, respectively.

$\left(C_{3}\right) \Rightarrow\left(C_{4}\right)$ follows by replacing $p+r, p-r, p+s, p-s$ in $\left(C_{3}\right)$ by $r, s, u, v$, respectively.

$\left(C_{4}\right) \Rightarrow\left(C_{6}\right)$ follows by replacing $r, s, u, v$ by $p-s, s, p-r, r$ or $s, p-s, r, p-r$ in $\left(C_{4}\right)$, respectively.

$\left(C_{6}\right) \Rightarrow\left(C_{7}\right)$ follows by taking $p=2$ with $r \geq 0$ in $\left(C_{6}\right)$.

$\left(C_{7}\right) \Rightarrow\left(C_{1}\right)$ follows by taking $s=1$ and $r=0$ in $\left(C_{7}\right)$.

$\left(C_{1}\right) \Leftrightarrow\left(C_{1}^{*}\right)$ is clear.

$\left(C_{4}\right) \Rightarrow\left(C_{5}\right)$ follows by letting $r+s=u+v=0, u=r, v=s$ and $Y=1$ in $\left(C_{4}\right)$. respectively.

$\left(C_{5}\right) \Rightarrow\left(C_{3}\right)$ follows by replacing $|Z|$ and $|X|$ in $\left(C_{5}\right)$ by $|Z|(|X||Y|)^{p}$ and $|X||Y|^{-1}$, 
$\left(C_{3}\right) \Rightarrow\left(C_{8}\right)$. Replacing $|X|$ by $|X|^{u}$ and $|Y|$ by $|Y|^{v}$ in $\left(C_{3}\right)$ and changing appropriately the notation for the exponents, we obtain $\left(C_{8}\right)$.

$\left(C_{8}\right) \Rightarrow\left(C_{3}\right)$ is clear.

To complete our proof of equivalence of all inequalities in this theorem and in Lemma 2.1, it suffices to show further the following implications.

$\left(E_{11}\right) \Rightarrow\left(J_{1}\right)$ follows by taking $f=G_{2} G_{1}^{-1}$ in $\left(E_{11}\right)$.

$\left(J_{1}\right) \Rightarrow\left(L_{3}\right)$ : Let $G_{1}(Y)=|Y|^{r_{1}}, G_{2}(Y)=|Y|^{r_{2}}$, where $r_{2} / r_{1}>1$ (hence $r_{2}>r_{1}>0$ or $\left.r_{2}<r_{1}<0\right)$. Then it follows from $\left(J_{1}\right)$ that $|E Y|^{r_{2} / r_{1}} \leq E|Y|^{r_{2} / r_{1}}$. Setting $Y=|X|^{r_{1}}$, we obtain $\left(L_{3}\right)$, see [14, page 162$]$.

$\left(E_{11}\right) \Rightarrow\left(J_{2}\right)$ follows by taking $f(x)=e^{t x}$ in $\left(E_{11}\right)$.

$\left(J_{2}\right) \Rightarrow\left(E_{1}\right)$ follows by taking $t=1$ and replacing $X$ by $\ln X$ in $\left(J_{2}\right)$.

Remark 2.3. Letting $r=p, s=p-1+h, u=p+h, v=p-1$ and $Y=Z=1$ with $h \geq 0$ and $p \in \mathbb{R}$ in $\left(C_{4}\right)$, we obtain the inequality (5) of [18]:

$$
E\left(|X|^{p-1+h}\right) E\left(|X|^{p}\right) \leq E\left(|X|^{p+h}\right) E\left(|X|^{p-1}\right) .
$$

That is,

$$
r(p):=\frac{E\left(|X|^{p-1}\right)}{E\left(|X|^{p}\right)}
$$

is a decreasing function of Sclove et al. [18] proved this property by means of the convexity of $f(t)=\ln E\left(|X|^{t}\right)$, see [14]. Clearly, our method is simpler than theirs.

Remark 2.4. Each $H_{i}$ (or $H_{i}^{*}$ ) is called Hölder's inequality, each $\left(C_{i}\right)$ (or $\left(C_{i}^{*}\right)$ ) is called CBS inequality, each $L_{i}$ is called Lyapunov's inequality, each $R_{i}$ is called Radon's inequality, each $\left(J_{i}\right)$ is related to Jensen's inequality.

\section{Acknowledgments}

The authors wish to thank three reviewers for their valuable suggestions that lead to substantial improvement of this paper. This work is dedicated to Professor Haruo Murakami on his 80th birthday.

\section{References}

[1] C. A. Infantozzi, "An introduction to relations among inequalities," in Proceedings of the American Mathematical Society Meeting 700, Cleveland, Ohio, USA, 1979.

[2] C. A. Infantozzi, "An introduction to relations among inequalities," Notices of the American Mathematical Society, vol. 141, pp. A918-A820, 1972.

[3] A. W. Marshall and I. Olkin, Inequalities: Theory of Majorization and Its Applications, vol. 143 of Mathematics in Science and Engineering, Academic Press, New York, NY, USA, 1979.

[4] L. Maligranda, "Why Hölder's inequality should be called Rogers' inequality," Mathematical Inequalities Eamp; Applications, vol. 1, no. 1, pp. 69-83, 1998.

[5] L. Maligranda, "Equivalence of the Hölder-Rogers and Minkowski inequalities," Mathematical Inequalities Eamp; Applications, vol. 4, no. 2, pp. 203-207, 2001.

[6] E. F. Beckenbach and R. Bellman, Inequality, Springer, Berlin, Germany, 4th edition, 1984.

[7] G. Casella and R. L. Berger, Statistical Inference, Duxbury Advanced Series, Thomson Learning, Duxbury, Mass, USA, 2002. 
[8] D. K. Callebaut, "Generalization of the Cauchy-Schwarz inequality," Journal of Mathematical Analysis and Applications, vol. 12, no. 3, pp. 491-494, 1965.

[9] K. L. Chung, A Course in Probability Theory, vol. 2 of Probability and Mathematical Statistics, Academic Press, New York, NY, USA, 2nd edition, 1974.

[10] J. Gurland, "Inequalities of expectations of random variables derived by monotonicity or convexity," The American Statistician, vol. 22, no. 2, pp. 26-27, 1968.

[11] G. H. Hardy, J. E. Littlewood, and G. Pólya, Inequalities, Cambridge University Press, Cambridge, UK, 2nd edition, 1952.

[12] M. Kendall and A. Stuart, The Advanced Theory of Statistics Vol 1, Macmillan, New York, NY, USA, 4th edition, 1977.

[13] Y.-C. Li and S.-Y. Shaw, "A proof of Hölder's inequality using the Cauchy-Schwarz inequality," Journal of Inequalities in Pure and Applied Mathematics, vol. 7, no. 2, 3 pages, 2006.

[14] M. Loève, Probability Theory, Springer, New York, NY, USA, 3rd edition, 1998.

[15] H. W. McLaughlin and F. T. Metcalf, "Remark on a recent generalization of the Cauchy-Schwarz inequality," Journal of Mathematical Analysis and Applications, vol. 18, no. 3, pp. 522-523, 1967.

[16] D. S. Mitrinović, Analytic Inequalities, Springer, New York, NY, USA, 1970.

[17] K. Mullen, "A note on the ratio of two independent random variables," The American Statistician, vol. 21, no. 3, pp. 30-31, 1967.

[18] S. L. Sclove, G. Simons, and J. V. Ryzin, "Further remarks on the expectation of the reciprocal of a positive random variable," The Americam Statistician, vol. 21, no. 4, pp. 33-34, 1967.

[19] X. F. Zong, "A further generalization of the Cauchy inequality," Journal of Qufu Normal University, vol. 26, no. 4, pp. 32-34, 2000 (Chinese). 